\title{
Application of a long-range forecasting model to earthquakes in the Japan mainland testing region
}

\author{
David A. Rhoades \\ GNS Science, P.O. Box 30-368, Lower Hutt 5040, New Zealand \\ (Received April 12, 2010; Revised August 6, 2010; Accepted August 10, 2010; Online published March 4, 2011)
}

\begin{abstract}
The Every Earthquake a Precursor According to Scale (EEPAS) model is a long-range forecasting method which has been previously applied to a number of regions, including Japan. The Collaboratory for the Study of Earthquake Predictability (CSEP) forecasting experiment in Japan provides an opportunity to test the model at lower magnitudes than previously and to compare it with other competing models. The model sums contributions to the rate density from past earthquakes based on predictive scaling relations derived from the precursory scale increase phenomenon. Two features of the earthquake catalogue in the Japan mainland region create difficulties in applying the model, namely magnitude-dependence in the proportion of aftershocks and in the Gutenberg-Richter $b$-value. To accommodate these features, the model was fitted separately to earthquakes in three different target magnitude classes over the period 2000-2009. There are some substantial unexplained differences in parameters between classes, but the time and magnitude distributions of the individual earthquake contributions are such that the model is suitable for three-month testing at $M \geq 4$ and for one-year testing at $M \geq 5$. In retrospective analyses, the mean probability gain of the EEPAS model over a spatially smoothed seismicity model increases with magnitude. The same trend is expected in prospective testing. The Proximity to Past Earthquakes (PPE) model has been submitted to the same testing classes as the EEPAS model. Its role is that of a spatially-smoothed reference model, against which the performance of time-varying models can be compared.
\end{abstract}

Key words: Statistical seismology, earthquake forecasting, Japan.

\section{Introduction}

The Every Earthquake a Precursor According to Scale (EEPAS) model (Rhoades and Evison, 2004) is an earthquake forecasting method based on the precursory scale increase $(\Psi)$ phenomenon (Evison and Rhoades, 2001, 2002, 2004) and associated predictive scaling relations-linear regressions of mainshock magnitude, logarithm of precursor time and logarithm of precursor area on precursor magnitude (Fig. 1). In this model, the problem of identifying the $\Psi$-phenomenon before the occurrence of a major earthquake is set aside, and each earthquake is regarded as a precursor of larger earthquakes to follow it in the longterm. The EEPAS model has previously been applied to a wider Japan region, including seismically active offshore zones, in forecasting earthquakes of magnitude $M>6.75$, using parameters estimated from the New Zealand earthquake catalogue, and then fitted to the wider Japan region at $M>6.25$ (Rhoades and Evison, 2005). It has also been fitted to the Kanto region in separate depth layers at $M>4.75$ (Rhoades and Evison, 2006). These studies indicate that the EEPAS model is an effective tool for forecasting the larger earthquakes in a catalogue, giving a measurable improvement over spatially-varying but time-invariant estimates of earthquake occurrence. Imoto and Rhoades

Copyright (C) The Society of Geomagnetism and Earth, Planetary and Space Sciences (SGEPSS); The Seismological Society of Japan; The Volcanological Society of Japan; The Geodetic Society of Japan; The Japanese Society for Planetary Sciences; TERRAPUB.

doi:10.5047/eps.2010.08.002
(2010) applied the EEPAS model together with other predictive parameters - the Gutenberg-Richter $a$ - and $b$-values and the change in $b$-value-to produce an even more informative model for the Kanto region.

The Collaboratory for the Study of Earthquake Predictability (CSEP) earthquake forecasting experiment in Japan presents an opportunity to apply the EEPAS model at a lower magnitude threshold than previously, and to have it formally and independently tested against other competing models. The $\Psi$ scaling relation between magnitude and precursor time (Evison and Rhoades, 2004) indicates that it might be possible to use the model either for three-month forecasts at $M \geq 4.0$ or for annual forecasts at $M \geq 5.0$. The model uses the minor earthquakes to forecast the major events, and requires catalogue completeness at nearly two orders of magnitude below the target magnitude threshold. With the current dense network of seismograph stations covering the Japan mainland region, the magnitude threshold of completeness is likely to be sufficiently low in recent years for fitting the model at $M \geq 4.0$. This suggests that it may be possible to enter the EEPAS model into the CSEP tests in the Japan mainland region for both three-month and one-year forecasts, but first it must be adapted to the testing region.

\section{Model Description}

The following is a brief description of the EEPAS model rate density. For a fuller explanation, see Rhoades and Evison (2004). The rate density $\lambda(t, m, x, y)$ of earthquake 
occurrence is defined for any time $t$, magnitude $m$ and location $(x, y)$, where $m$ exceeds a threshold magnitude $m_{\mathrm{c}}$, and $(x, y)$ is a point in a region of surveillance $R$. Each earthquake $\left(t_{i}, m_{i}, x_{i}, y_{i}\right)$, with $m_{i}$ exceeding a minimum magnitude threshold $m_{0}$, contributes a transient increment $\lambda_{i}(t, m, x, y)$ to the future rate density in its vicinity, given by

$$
\lambda_{i}(t, m, x, y)=w_{i} f_{1 i}(t) g_{1 i}(m) h_{1 i}(x, y)
$$

where $w_{i}$ is a weighting factor that may depend on other earthquakes in the vicinity, and $f_{1 i}, g_{1 i}$ and $h_{1 i}$ are densities of the probability distributions for time, magnitude and location, respectively. Based on the $\Psi$ predictive relation for mainshock magnitude (Fig. 1(a)), the magnitude density $g_{1 i}$ takes the form

$$
g_{1 i}(m)=\frac{1}{\sigma_{M} \sqrt{2 \pi}} \exp \left[-\frac{1}{2}\left(\frac{m-a_{M}-b_{M} m_{i}}{\sigma_{M}}\right)^{2}\right]
$$

where $a_{M}, b_{M}$ and $\sigma_{M}$ are parameters. Based on the $\Psi$ predictive relation for precursor time (Fig. 1(b)), the time density $f_{1 i}$ takes the form

$$
\begin{aligned}
f_{1 i}(t)= & \frac{H\left(t-t_{i}\right)}{\left(t-t_{i}\right) \sigma_{T} \ln (10) \sqrt{2 \pi}} \\
& \times \exp \left[-\frac{1}{2}\left(\frac{\log \left(t-t_{i}\right)-a_{T}-b_{T} m_{i}}{\sigma_{T}}\right)^{2}\right]
\end{aligned}
$$

where $H(s)=1$ if $s>0$ and 0 otherwise, and $a_{T}, b_{T}$ and $\sigma_{T}$ are parameters. Based on the $\Psi$ predictive relation for precursor area (Fig. 1(c)), the location density $h_{1 i}$ takes the form

$$
\begin{aligned}
h_{1 i}(x, y)= & \frac{1}{2 \pi \sigma_{A}^{2} 10^{b_{A} m_{i}}} \\
& \times \exp \left[-\left(\frac{\left(x-x_{i}\right)^{2}+\left(y-y_{i}\right)^{2}}{2 \sigma_{A}^{2} 10^{b_{A} m_{i}}}\right]\right.
\end{aligned}
$$

where $\sigma_{A}$ and $b_{A}$ are parameters. The total rate density is obtained by summing over all past occurrences, including earthquakes outside $R$, which could affect the rate density within $R$ :

$$
\begin{aligned}
\lambda(t, m, x, y)= & \mu \lambda_{0}(t, m, x, y) \\
& +\sum_{t \geq t_{0} ; m_{i} \geq m_{0}} \eta\left(m_{i}\right) \lambda_{i}(t, m, x, y)
\end{aligned}
$$

where $\mu$ is a parameter, $\lambda_{0}$ is the rate density of the Proximity to Past Earthquakes (PPE) model described in the next paragraph, $t_{0}$ is the time of the beginning of the catalogue and $\eta$ is a normalizing function, given by

$$
\begin{aligned}
\eta(m)= & \frac{b_{M}(1-\mu)}{\bar{w}} \\
& \times \exp \left[-\beta\left(a_{m}+\left(b_{M}-1\right) m+\frac{\sigma_{M}^{2} \beta}{2}\right)\right],
\end{aligned}
$$

where $\beta=b \ln 10, b$ is the Gutenberg-Richter $b$-value, and $\bar{w}$ is the average of the relevant earthquake weights. A further adjustment is made to the rate density to compensate for the missing contribution from earthquakes below the magnitude threshold $m_{0}$ (Rhoades and Evison, 2004).
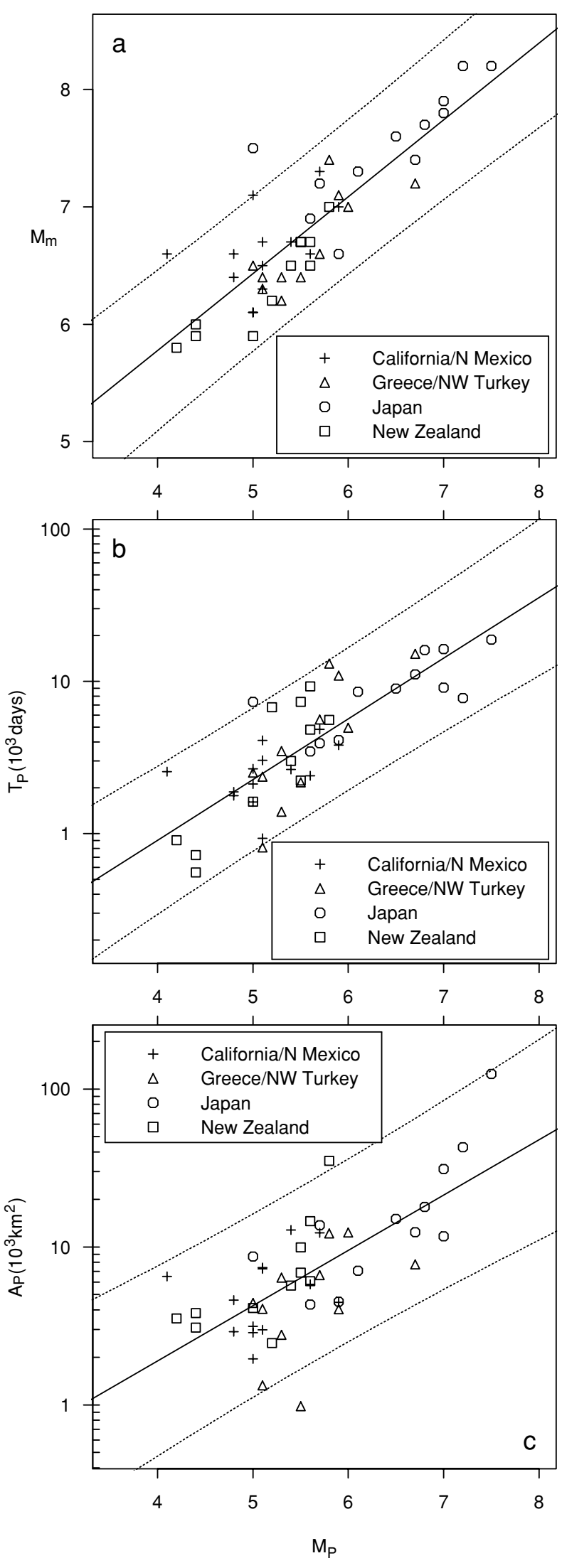

Fig. 1. Predictive relations and 95\% tolerance limits, derived from 47 examples of the $\Psi$-phenomenon in four well-catalogued regions: (a) Mainshock magnitude $M_{m}$ versus precursor magnitude $M_{P}$; (b) Precursor time $T_{P}$ versus $M_{P}$; Precursor area $A_{P}$ versus $M_{P}$. After Evison and Rhoades (2004). 
The PPE model (Rhoades and Evison, 2004, 2005) was fashioned from a model proposed by Jackson and Kagan (1999). The rate density $\lambda_{0}$ has the form

$$
\lambda_{0}(t, m, x, y)=f_{0}(t) g_{0}(m) h_{0}(t, x, y)
$$

where

$$
\begin{gathered}
f_{0}(t)=\frac{1}{t-t_{0}}, \\
g_{0}(m)=\beta \exp \left[-\beta\left(m-m_{\mathrm{c}}\right)\right] \quad\left(m \geq m_{0}\right)
\end{gathered}
$$

and $h_{0}(t, x, y)$ is the sum, over all earthquakes with $m_{i}>$ $m_{\mathrm{c}}$ from time $t_{0}$ up to, but not including, time $t$, of smoothing kernels of the form

$$
h_{0 i}\left(r_{i}\right)=a\left(m_{i}-m_{\mathrm{c}}\right) \frac{1}{\pi}\left(\frac{1}{d^{2}+r_{i}^{2}}\right)+s .
$$

In Eq. (10), $r_{i}$ is the distance in $\mathrm{km}$ between $(x, y)$ and the epicentre $\left(x_{i}, y_{i}\right)$ of the $i$ th precursory earthquake; and $a, d$ and $s$ are constant parameters.

Rhoades and Evison (2004) proposed two different weighting strategies: equal weighting of all earthquakes, and down-weighting of aftershocks. The weights in the latter strategy are derived from the rate densities, $\lambda_{0}$ and $\lambda^{\prime}$, of a smoothed seismicity baseline model (PPE) and a model incorporating aftershocks, respectively:

$$
w_{i}=\frac{v \lambda_{0}\left(t_{i}, m_{i}, x_{i}, y_{i}\right)}{\lambda^{\prime}\left(t_{i}, m_{i}, x_{i}, y_{i}\right)} .
$$

The aftershock model incorporates epidemic-type aftershock behaviour (Ogata, 1989, 1998; Console and Murru, 2001), and is designed to admit as aftershocks only earthquakes that are close to the mainshock source and much smaller than the mainshock. The rate density $\lambda^{\prime}$ is of the form

$$
\lambda^{\prime}(t, m, x, y)=v \lambda_{0}(t, m, x, y)+\kappa \sum_{t \geq t_{0}} \lambda_{i}^{\prime}(t, m, x, y)
$$

where $\lambda_{0}$ is as above; $v$ and $\kappa$ are constant parameters; and

$$
\lambda_{i}^{\prime}=f_{2 i}(t) g_{2 i}(m) h_{2 i}(x, y) .
$$

Here, $f_{2 i}, g_{2 i}$ and $h_{2 i}$ are functions for the time, magnitude and location of the aftershocks of the $i$ th earthquake. The time distribution follows the Omori-Utsu law (e.g. Ogata, 1983); the magnitude distribution has regard to the Gutenberg-Richter law and Bäth's law (Bäth, 1965); and the location distribution is bivariate normal with circular symmetry and has regard to Utsu's areal relation (Utsu, 1961). Thus

$$
\begin{gathered}
f_{2 i}(t)=H\left(t-t_{i}\right) \frac{p-1}{\left(t-t_{i}+c\right)^{p}}, \\
g_{2 i}(m)=H\left(m_{i}-\delta-m\right) \beta \exp \left[-\beta\left(m-m_{i}\right)\right],
\end{gathered}
$$

and

$$
h_{2 i}(x, y)=\frac{1}{2 \pi \sigma_{U}^{2}} \exp \left[-\frac{\left(x-x_{i}\right)^{2}+\left(y-y_{i}\right)^{2}}{2 \sigma_{U}^{2} 10^{m_{i}}}\right]
$$

where $c, p, \delta$ and $\sigma_{U}$ are constant parameters, $\beta$ is as in Eq. (6), and the step function $H$ is as in Eq. (3).

\section{Application to the Japan Mainland Region}

The region of surveillance $R$ considered here is the Japan mainland region as defined by the Japan Earthquake Forecast Testing Center (Fig. 2). In order to forecast earthquakes of $M>3.95$ for earthquakes at hypocentral depths $h \leq 30 \mathrm{~km}$ in this region, we make use of the earthquakes in a wider region delimited by the outer polygon in Fig. 2. The catalogue used is the Japan Meteorological Agency (JMA) catalogue, supplied by the Testing Center, covering the period 1 January 1965 to 31 January 2009. Our analysis is restricted to earthquakes of magnitude $M>2.15$.

It is necessary to understand the varying completeness of the catalogue with time before applying the EEPAS model. The catalogue is expected to be complete for $M \geq 4$ for the whole period, but to show a decreasing magnitude of completeness due to improvement of the seismograph network over the period. Figure 3 is a plot of the ratio of the number of earthquakes $N(M>m+0.5)$ exceeding the magnitude threshold $m+0.5$ to the number $N(M>m)$ exceeding the threshold $m$, for values of $m$ ranging from 2.15 to 3.65 . For a catalogue complete above magnitude $m$, the expected value of this ratio is $10^{0.5 b}$, where $b$ is the Gutenberg-Richter $b$-value. The expected value for a $b$ value of 1 is plotted in Fig. 3 for comparison with the data. The ratio for $m=3.65$ is rather constant over the period at about 0.4 , corresponding to a $b$-value of about 0.8 . The ratios for $m=2.65$ and $m=3.15$ start at a high value and show a decreasing trend, indicative of improving completeness, up until 1990, and are constant at about 0.4 thereafter. The curve for $m=2.15$ also starts at a high value and decreases until 2000, after which it settles at the same level as the other curves. It appears therefore that the catalogue is satisfactorily complete for $M>2.15$ since about 2000 .

The PPE and aftershock models were used to compute weights for earthquakes in $R$ using Eq. (11), with PPE model parameters fitted to the present data set and standard values of the aftershock model parameters from previous studies (Rhoades and Evison, 2004) as listed in Table 1. Although the aftershock parameters are expected to vary from region to region, it was not considered necessary to optimise them for the present test region, because it is only the weights derived from them that are used in the EEPAS model. Most of the weights are close to 0 or 1 and are not sensitive to small changes in the aftershock parameters. Also, optimising the aftershock model would not ensure that the weights would be optimal for the EEPAS model. It is not practicable to fit the aftershock parameters to optimise the EEPAS model itself, because iterative recomputation of the weights is too time-consuming.

The weight associated with a given earthquake can be interpreted as the probability that it is an independent event, as opposed to an aftershock of a previous event (Zhuang et al., 2002). Therefore the mean weight of a class of earthquakes represents the proportion $p_{I}$ of earthquakes in the class which are independent events. Figure 4 shows the mean weight of earthquakes with magnitude $M>m$ in $R$ over the period 2000-2009, as a function of the magnitude threshold $m$. It shows that there is significant variation of $p_{I}$ with $m$. The left panel shows that $p_{I}$ decreases from 0.28 at $m=2.2$ to 0.22 at $m=2.8$, and then increases to 0.34 
Table 1. Parameters of the PPE and EEPAS models, as set or optimised for the Japan mainland region over the period 2000-2009, and used for testing of these models in the Japan Earthquake Forecast Testing Center.

\begin{tabular}{|c|c|c|c|c|}
\hline \multicolumn{5}{|c|}{ Magnitude class } \\
\hline & $3.95<M<4.45$ & $3.95<M<4.45$ & $3.95<M<4.45$ & \multirow[b]{2}{*}{ Restriction } \\
\hline Parameter & & Value & & \\
\hline \multicolumn{5}{|c|}{ Aftershock model } \\
\hline$c$ & $0.03 \mathrm{~d}$ & $0.03 \mathrm{~d}$ & $0.03 \mathrm{~d}$ & fixed \\
\hline$p$ & 1.2 & 1.2 & 1.2 & fixed \\
\hline$\sigma_{U}$ & 0.0056 & 0.0056 & 0.0056 & fixed \\
\hline$\kappa$ & 0.09 & 0.09 & 0.09 & fixed \\
\hline$v$ & 0.75 & 0.75 & 0.75 & fixed \\
\hline$\delta$ & 0.7 & 0.7 & 0.7 & fixed \\
\hline \multicolumn{5}{|c|}{ PPE model } \\
\hline$b$ & 1.0 & 1.0 & 1.0 & See text \\
\hline$a$ & 0.30 & 0.35 & 0.28 & $\geq 0$ \\
\hline$d$ & $1.0 \mathrm{~km}$ & $1.0 \mathrm{~km}$ & $1.0 \mathrm{~km}$ & $\geq 1$ \\
\hline$s$ & $1.51 \times 10^{-11}$ & $1.37 \times 10^{-11}$ & $1.64 \times 10^{-11}$ & $\geq 0$ \\
\hline \multicolumn{5}{|c|}{ EEPAS model } \\
\hline$b_{\text {EEPAS }}$ & 0.70 & 0.70 & 0.80 & See text \\
\hline$a_{M}$ & 1.0 & 1.0 & 1.0 & $1.0-2.0$ \\
\hline$b_{M}$ & 1.0 & 1.0 & 1.0 & fixed \\
\hline$\sigma_{M}$ & 0.15 & 0.16 & 0.31 & $0.1-0.6$ \\
\hline$a_{T}$ & 2.14 & 2.20 & 1.30 & $1.0-2.5$ \\
\hline$b_{T}$ & 0.37 & 0.31 & 0.41 & $0.3-0.6$ \\
\hline$\sigma_{T}$ & 0.15 & 0.15 & 0.12 & $0.1-0.6$ \\
\hline$b_{A}$ & 0.30 & 0.35 & 0.37 & $0.2-0.6$ \\
\hline$\sigma_{A}$ & 4.01 & 2.33 & 2.39 & $0.5-30$ \\
\hline$\mu$ & 0.06 & 0.09 & 0.05 & $0-1$ \\
\hline $\bar{w}$ & 0.21 & 0.21 & 0.37 & See text \\
\hline
\end{tabular}

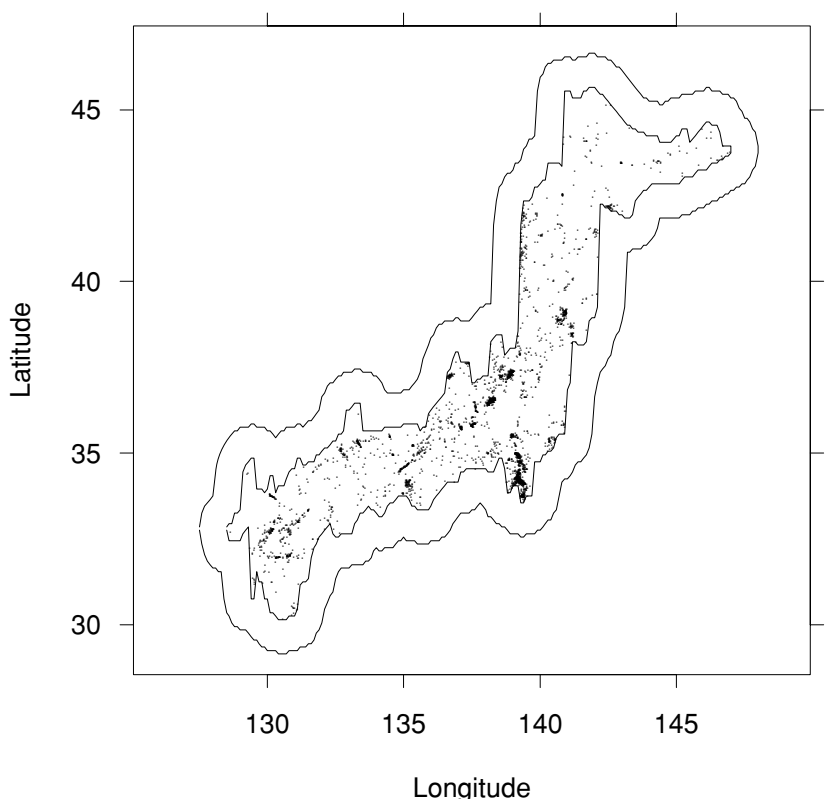

Fig. 2. Japan mainland region of surveillance $R$ (inner polygon), data collection region (outer polygon) used for fitting of models, and epicentres of $M>3.95$ earthquakes in the period 2000-2009.

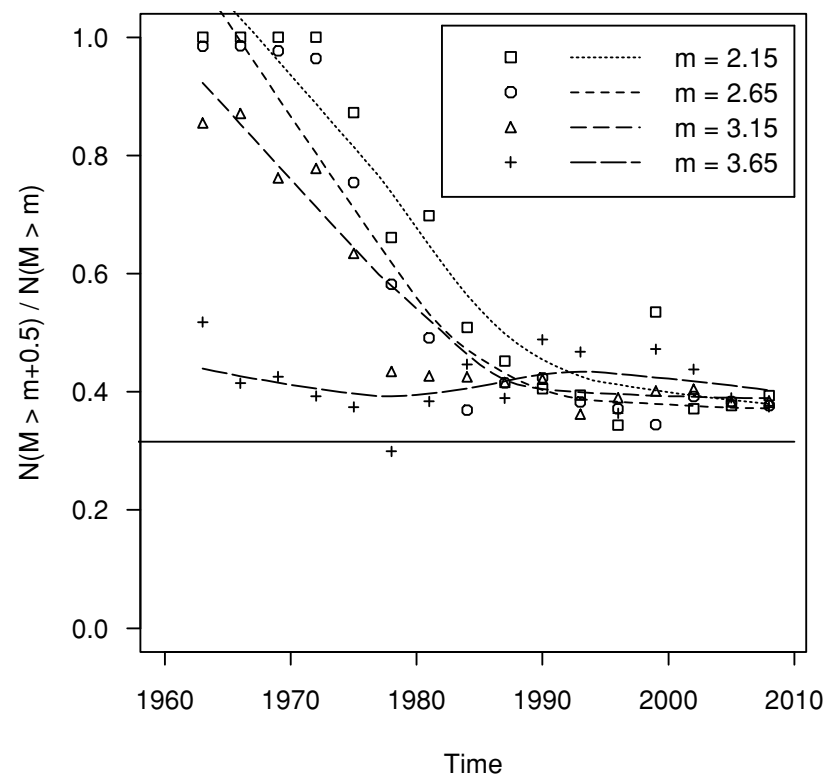

Fig. 3. Ratio of numbers of earthquakes $N(M>m+0.5) / N(M>m)$ in three year intervals and smooth local regression fits. The solid line shows the expected value of the ratio under catalogue completeness and a Gutenberg-Richter $b$-value of 1 . at $m=5.0$. The right panel shows that, as $m$ is increased above $5.0, p_{I}$ increases rapidly and is 1 for $m \geq 6.2$.

The mean weights plotted in Fig. 4 are generally lower and more strongly dependent on $m$ than in previous cata- logues to which EEPAS has been applied. For example, Rhoades (2009) reported $p_{I}$ values of 0.71 for California and 0.83 for the Kanto region at $m=5.0$. This magnitude dependence is problematical for the EEPAS model, 


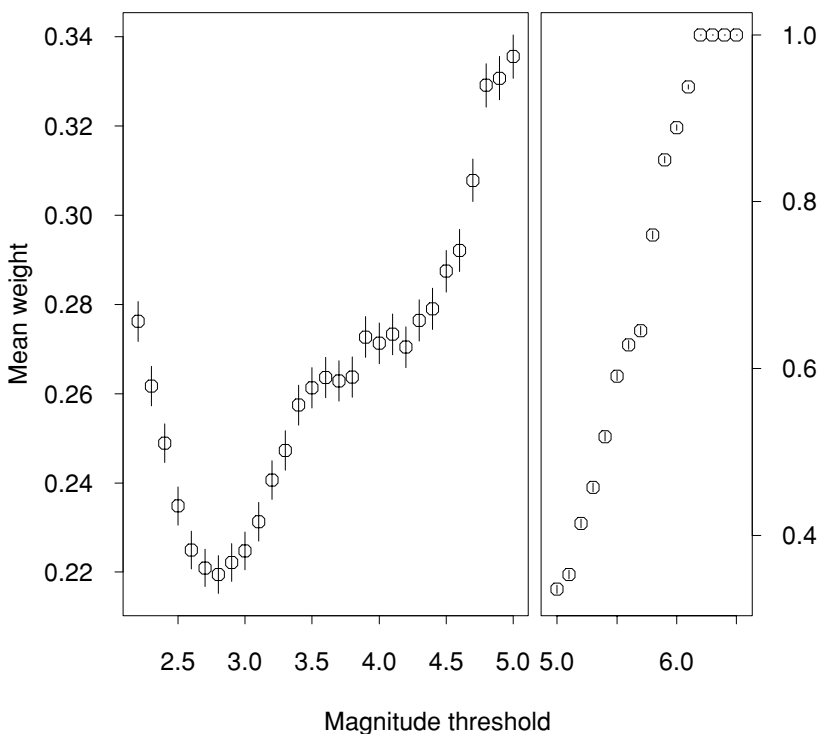

Fig. 4. Mean weight (Eq. (11)) of earthquakes in Japan mainland region of surveillance over the period 2000-2009 as a function of lower magnitude threshold.

which implicitly assumes that $p_{I}$ is independent of magnitude. The same assumption was made in a version of EEPAS allowing explicitly for aftershocks of forecasted events (Rhoades, 2009). Deviation of the data from this assumption will undoubtedly affect the fit and performance of the standard EEPAS model applied here, which is the only version so far adapted for CSEP testing. The situation is complicated further by the fact that the dependence of $p_{I}$ on $m$ is bound to be somewhat time dependent, if only because the maximum magnitude in $R$ will differ in different time periods. The maximum magnitude contributing to Fig. 4 is 7.3; a very large earthquake in $R$ with $M>8$ would be expected to have numerous aftershocks with $M>6$, which would have the effect of reducing $p_{I}$ for $m \geq 6$ to lower values than are shown in Fig. 4.

The Gutenberg-Richter $b$-value was estimated using the maximum-likelihood method for earthquakes in $R$ over the period 2000-2009, for a range of magnitude thresholds between $m=2.2$ and $m=5.0$ (Fig. 5). Two different $b$-value estimates are made — a standard (unweighted) estimate, and a weighted estimate in which each earthquake is given the weight $w_{i}$ computed by Eq. (11) - in order to accommodate the two different weighting options in the EEPAS model. Figure 5 shows that these two estimates differ significantly, with the unweighted estimate being higher except for $m \leq 2.5$. The estimates also vary with $m$, in a manner which is obviously partially confounded with the variation of mean weight with $m$ (Fig. 4). A side-effect of this variation is that straightforward estimates of $b$-value above, say, the minimum magnitude $m_{0}=2.15$, may not work well in the EEPAS model. However, a single value must be chosen, because the model makes no allowance for the magnitude dependence seen in Figs. 4 and 5.

The EEPAS model is primarily designed to forecast the major earthquakes, rather than their aftershocks. The dependence of $b$ and $p_{I}$ on the magnitude threshold precip-

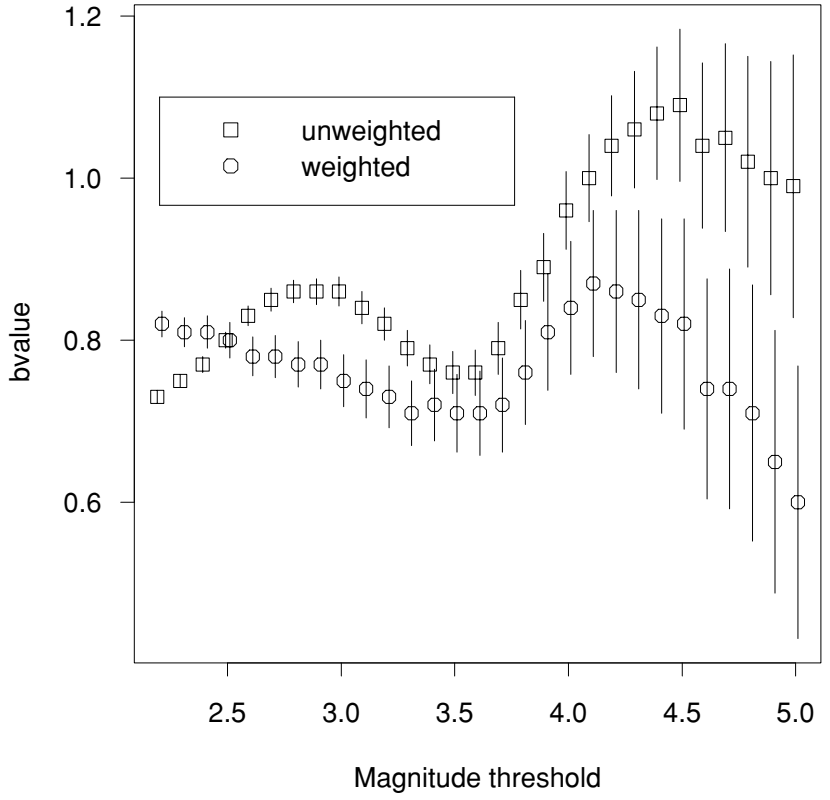

Fig. 5. Unweighted and weighted maximum likelihood estimates of Gutenberg-Richter $b$-value for earthquakes in the Japan mainland region of surveillance over the period 2000-2009 as a function of lower magnitude threshold. Error bars are \pm 2 standard errors. Weights are calculated using Eq. (11).

itated a decision to fit both the PPE and EEPAS models within restricted target magnitude classes, namely $3.95<$ $M<4.45,4.45<M<4.95$, and $4.95<M<9.05$, so that the performance at higher magnitudes is not adversely affected by fitting the model to a much larger number of predominantly smaller events.

\subsection{Fitted parameters}

The EEPAS model was found to fit all three target magnitude classes better with aftershocks down-weighted than with equal weights. The optimal parameters for both models and all three magnitude classes are listed in Table 1. Note that the PPE and EEPAS models use different Gutenberg-Richter $b$-values. The PPE model $b$-value (" $b$ " in Table 1) is needed for magnitude scaling of earthquakes within the target magnitude range (Eq. (9)). It is estimated from the unweighted $b$-value for magnitudes in the target range. For the EEPAS model, the $b$-value (" $b_{\text {EEPAS" }}$ in Table 1) plays a role in the normalising function (Eq. (6)), that is needed to retain an appropriate relation between the number of "small" precursory earthquakes and the number of "large" predicted events. For a given target magnitude range, it was arbitrarily set taking into account the weighted $b$-value pertaining to the most influential magnitudes of precursory earthquakes (Fig. 5). The parameter also plays a role in the normalising function. The value of this parameter was arbitrarily adjusted also for each magnitude class so that the number of earthquakes predicted by the EEPAS model approximately matched the actual number over the fitting period. The parameter $b_{M}$ was set to 1 , as in several previous applications of the EEPAS model (Rhoades and Evison, 2005, 2006; Rhoades, 2007), and thus a constant difference is assumed between the magnitude of a precursor and the mean magnitude of its contribution to the 

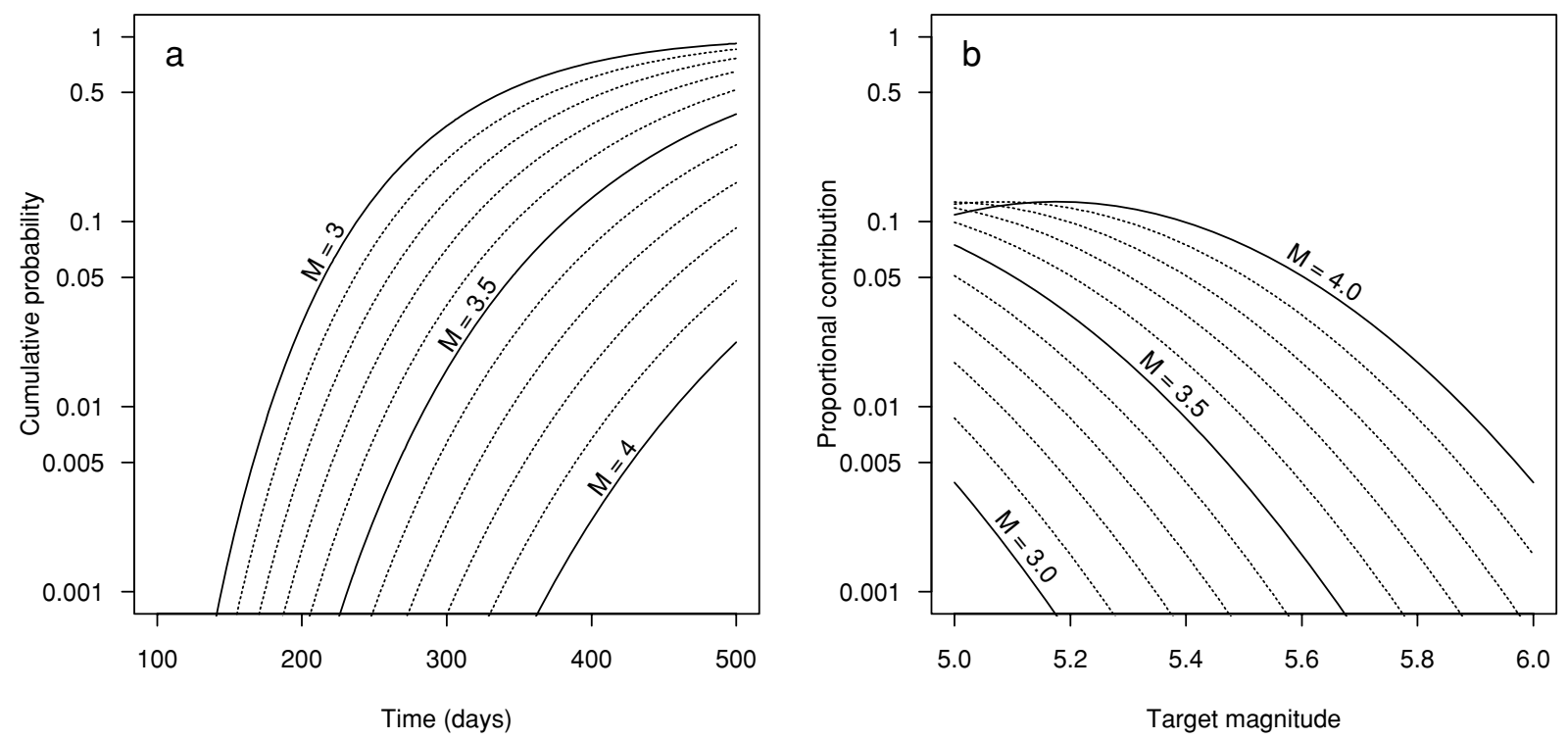

Fig. 6. Precursory earthquake contributions to EEPAS model rate density in the target magnitude range $4.95<M<9.05$, with parameters from Table 1. (a) Cumulative time distribution (Eq. (3)) in contribution from a precursory earthquake in the magnitude range 3-4, at intervals of 0.1, starting from its time of occurrence (b) Proportional contribution to the total rate density at a given target magnitude from precursors in the magnitude range $3-4$, at intervals of 0.1 .

rate density. The other parameters (three for PPE and eight for EEPAS) were optimised by maximum likelihood, within upper and lower bounds as indicated in Table 1. Such constraints are necessary, inter alia, to limit the distorting effect of aftershocks on the fitted parameters.

The parameters of the PPE model and several parameters of the EEPAS model do not vary appreciably across the three magnitude classes. In all cases, $a_{M}$ attained its minimum bound of 1.0; this rather low value may be an effect of the model compromising between forecasting major earthquakes and their aftershocks. This effect appears to be confirmed by the fitted values of $\mu(<0.1)$, which represents the proportion of unpredicted earthquakes in the EEPAS model. Such low values can be compared to the relatively high proportion $(>0.5)$ of aftershocks in each magnitude class, and indicate that the EEPAS model has adjusted itself to successfully forecast many of the aftershocks. The values of the parameters $a_{T}$ and $\sigma_{A}$ differ across the three magnitude classes for unknown reasons, but perhaps reflecting differing adjustments of the scales of precursor time and area to accommodate the aftershocks, or latent complexities in the scaling of precursor time and area which are not evident from Fig. 1. As an example of such complexities, it seems possible that time and area scaling could be affected locally by the average rate of earthquake occurrence (Rhoades, 2009). The values for the highest magnitude class are nearest to those found in previous studies of the Japan region.

The fitted values of $\sigma_{M}$ and $\sigma_{T}$ are generally lower than in previous applications of the model. A consequence of the low values of $\sigma_{M}$ is that the precursory earthquakes contributing to the rate density for target earthquakes of a given magnitude fall into a rather narrow magnitude range. For example, at a target magnitude of $M=4.0$, more than 99.9 percent of the contributions to the rate density come from earthquakes in the magnitude range $2.45<M<3$.45. Ev- idently, with these fitted parameters, the chosen minimum magnitude threshold of $m_{0}=2.15$ is more than adequate to ensure that all precursory earthquakes relevant to the target range are included in the analysis. This threshold could be raised to 2.45 with hardly any loss of information.

\subsection{Time and magnitude distributions of contributions to rate density}

Let us consider the time distribution of the contribution from an earthquake of $M 2.5$, which is at the lower end of the precursor magnitude range that could make an effective contribution to the forecast in the lowest targeted magnitude range. Using Eq. (3) and the parameters $a_{T}$ and $b_{T}$ from Table 1, the logarithm of the time in days, starting from the time of its occurrence, is normally distributed with mean 3.07 and standard deviation 0.15 . The cumulative distribution is less than $10^{-13}$ at 3 months after its occurrence. Therefore no earthquakes occurring during a three-month forecasting period would have any appreciable influence on the rate density before the end of the period. The same is true for contributions to the middle targeted magnitude range but not for those to the highest magnitude range, for which the parameter $a_{T}$ is lower.

Figure 6(a) shows the cumulative time distribution in the contribution from precursory earthquakes between magnitudes 3 and 4, at intervals of 0.1 , in the model for the highest target magnitude range $4.95<M<9.05$. This shows, for example, that for a precursor of magnitude 3 , about half of its contribution is made within one year of its occurrence, and that for a precursor of magnitude 4, only about one-thousandth of its contribution is made within one year. Figure 6(b) shows the overall proportional contribution to the total rate density at a given target magnitude from precursors in the same magnitude range. It shows, for example, the proportional contribution to the total rate density at the target magnitude of 5.0 that originates from precursors of magnitude 3.0 is about 0.004 , and the proportional contri- 


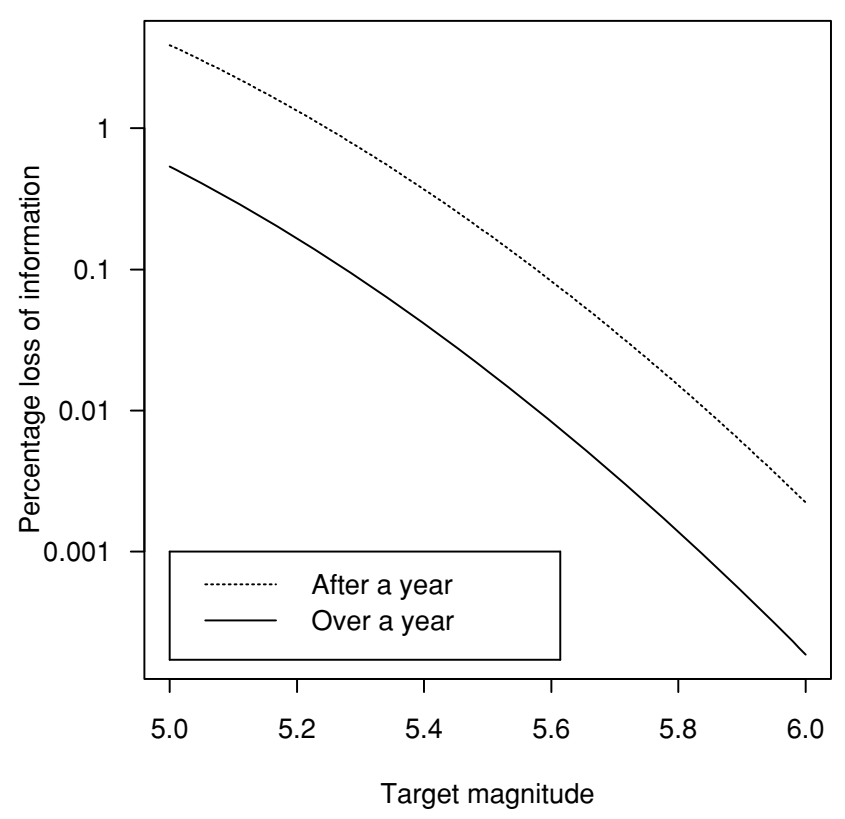

Fig. 7. Percentage loss of precursory information as a function of target magnitude for a one year forecast window due to non-inclusion in the forecast of earthquakes occurring during the year. Separate curves are shown for the loss at the end of the one year forecast window and the average loss over the whole year.

bution from precursors of magnitude 4.0 is about 0.1 .

When EEPAS is applied with a one-year forecasting window, there is a potential loss of information because earthquakes that occur during the forecasting window cannot contribute to the forecast. For a given target magnitude, this loss can be quantified by multiplying the time-distribution loss for each precursor magnitude (Fig. 6(a)) by the proportional contribution made by that precursor magnitude (Fig. 6(b)) and summing over precursor magnitude bins. The result is seen in Fig. 7, which shows the estimated instantaneous loss of information at the end of a one-year forecasting interval as well as the average loss over the whole year to which the forecast applies. The instantaneous loss at the end of the year is about 5 percent for a target magnitude of 5.0 and reduces rapidly to less than 0.01 percent for a target magnitude of 6.0. Averaged over the year of the forecast, the losses are trivial, ranging from 0.5 percent at $M 5.0$ to about 0.0002 percent at $M$ 6.0. It is clear therefore that the EEPAS model can be entered for CSEP testing in the one-year forecast class with hardly any loss of information due to the exclusion of precursory earthquakes.

\subsection{Goodness of fit and expected forecasting perfor- mance}

Goodness of fit is assessed here using the Akaike Information Criterion (AIC) statistic (Akaike, 1974), defined for a particular model $\mathrm{M}$ as

$$
\mathrm{AIC}_{\mathrm{M}}=-2 \ln L_{\mathrm{M}}+2 p_{\mathrm{M}}
$$

where $\ln L_{\mathrm{M}}$ is the optimised log likelihood of the model, and $p_{\mathrm{M}}$ is the number of fitted parameters. A relatively low value of AIC indicates a relatively high information value and a model which explains the data relatively well. We measure the information value of a fitted model by the information rate per earthquake, $I_{\mathrm{M}}$, defined (Harte and Vere-Jones, 2005; Rhoades and Gerstenberger, 2009) by

$$
I_{\mathrm{M}}=\left(\mathrm{AIC}_{\mathrm{SUP}}-\mathrm{AIC}_{\mathrm{M}}\right) /(2 N)
$$

where $N$ is the number of earthquakes in the target set, and where SUP is the best-fitting Stationary Uniform Poisson model with $b$-value 1 . The information rate thus compares each model to a model of least information, with no temporal or spatial variation in the earthquake occurrence rate density, although any other baseline model could equally well have been used.

For the purposes of the AIC statistic, the number of fitted parameters in the EEPAS, PPE, and SUP models is 9, 3 and 1, respectively. These numbers are less than the total number of parameters in the models, but represent the number of parameters that were adjusted in optimising the log likelihood. The effect of the number of fitted parameters on the information rates of the models is quite small because of the large number of earthquakes, $N$, in each of the target sets (Table 2).

The information rates of the PPE and EEPAS models for each magnitude class are given in Table 2. It is the difference $I_{\text {EEPAS }}-I_{\mathrm{PPE}}$ that is of most interest. This difference is seen to be $0.24,0.42$ and 1.02 for the three magnitude classes in increasing order. The geometric mean probability gain per earthquake is calculated as $\exp \left(I_{\mathrm{EEPAS}}-I_{\mathrm{PPE}}\right)$. This statistic is given in parenthesis in Table 2, and is seen to increase from 1.27 for the $3.95<M<4.45$ magnitude class to 2.77 for the $4.95<M<9.05$ magnitude class. The last value is towards the high end of probability gains found in previous applications of the EEPAS model. Although probability gains estimated from fitting are not necessarily a good guide to future performance, these values indicate that the model is likely to perform better at higher magnitudes than at lower magnitudes.

The effect on the information rate of fitting the model separately to different magnitude classes is substantial. For example, the EEPAS parameters fitted to the $3.95<M<$ 4.45 class give an information rate of only 1.43 when applied to the $4.95<M<9.05$ class. This is less than that of the PPE model, and corresponds to a probability gain of 0.74. Conversely, when the EEPAS parameters fitted to the $4.95<M<9.05$ class give an information rate of only 1.80 when applied the $3.95<M<4.45$ class. This is again less than that of the PPE model, and corresponds to a probability gain of 0.61 .

The approximate contribution that an individual target earthquake (with index $i$ ) makes to the probability gain of the EEPAS model over the PPE model is given by

$$
G_{i}=\left[\frac{\lambda_{1}\left(t_{i}, m_{i}, x_{i}, y_{i}\right)}{\lambda_{0}\left(t_{i}, m_{i}, x_{i}, y_{i}\right)}\right]\left(\frac{\hat{N}_{\mathrm{PPE}}}{\hat{N}_{\mathrm{EEPAS}}}\right)
$$

where $\hat{N}_{\mathrm{M}}$ is the expected number of target earthquakes under model M. This approximation was obtained by scaling the rate densities by their expected number of earthquakes and disregarding the small effect of the number of fitted parameters on the information score. Figure 8 shows $G_{i}$ plotted against magnitude $m_{i}$ (Fig. 8(a)) and weight $w_{i}$ 
Table 2. Information rate per earthquake, $I_{\mathrm{PPE}}$ and $I_{\mathrm{EEPAS}}$, of the PPE and EEPAS models relative to the SUP model, and (in parenthesis) geometric mean probability gain of EEPAS model over PPE model, for different targeted magnitude ranges.

\begin{tabular}{cccc}
\hline & \multicolumn{4}{c}{ Magnitude class (and number of target earthquakes) } \\
& $3.95<M<4.45$ & $4.45<M<4.95$ & $4.95<M<9.05$ \\
& $(N=1040)$ & $(N=396)$ & $(N=148)$ \\
Model & \multicolumn{2}{c}{ Information rate (and probability gain) } \\
\hline PPE & 2.26 & 2.25 & 1.74 \\
EEPAS & $2.50(1.27)$ & $2.67(1.52)$ & $2.76(2.77)$ \\
\hline
\end{tabular}
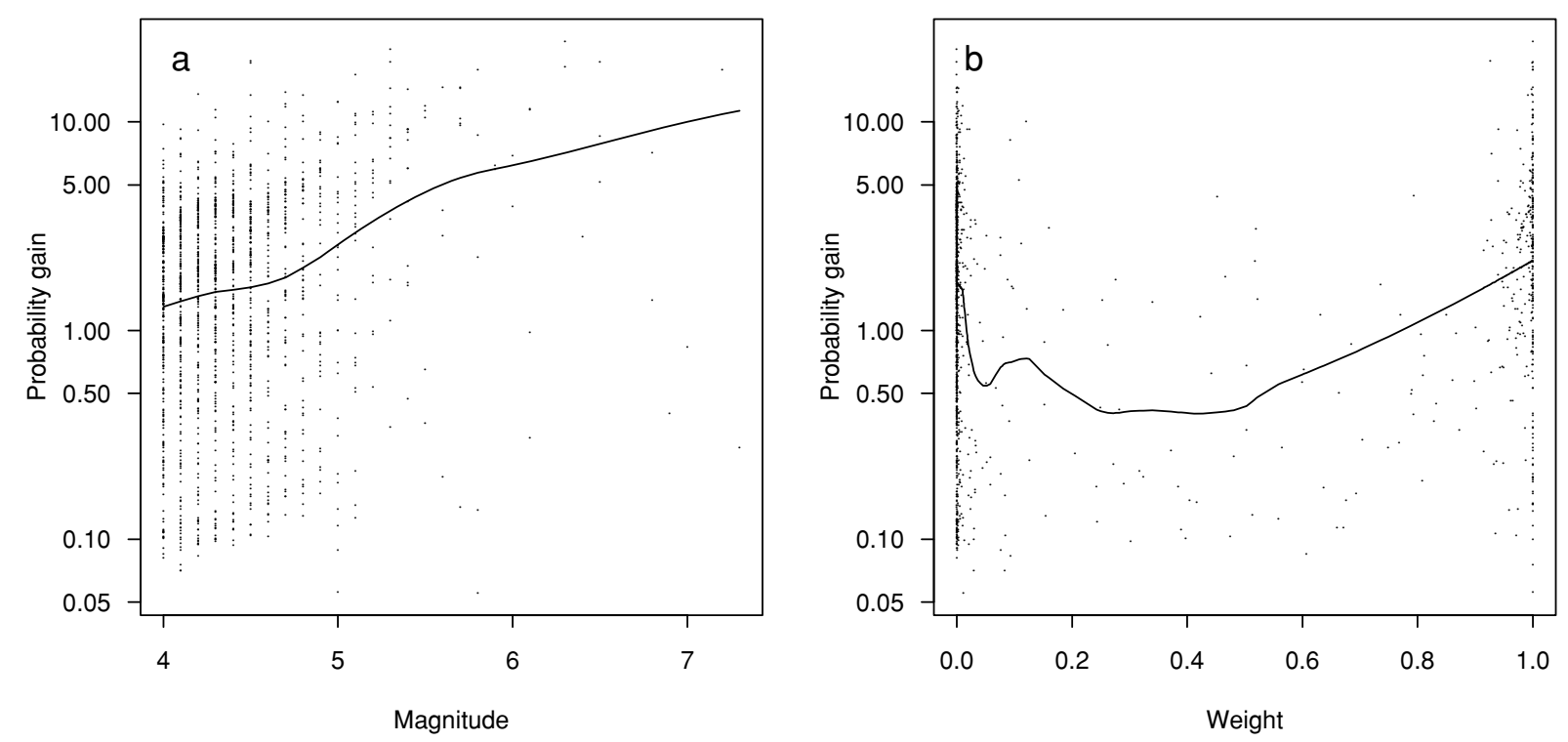

Fig. 8. Probability gain of EEPAS model over PPE model for individual target earthquakes in the Japan mainland region, 2000-2009, as a function of (a) magnitude and (b) weight. The robust smooth trend lines are computed using locally-weighted least squares.

(Fig. 8(b)). It confirms that there is a clear increasing trend in $G_{i}$ with magnitude as shown by the robust smooth trend line in Fig. 8(a), although the probability gain for individual earthquakes varies over a wide range between 0.05 and 20 . The trend of probability gain against weight (Fig. 8(b)) is not so straightforward, but the average probability gain for fully independent earthquakes $\left(w_{i}=1\right)$ is 2.3 , while that for pure aftershocks $\left(w_{i}=0\right)$ is 1.7 . Thus, although the EEPAS model is better at forecasting independent earthquakes than aftershocks, it nevertheless has some success in forecasting aftershocks. This confirms that, in the optimisation of the parameters, a compromise has been fashioned between forecasting main shocks, as the model is intended to do, and forecasting aftershocks, which are predominant in most of the target magnitude range. The present implementation of the model therefore deviates somewhat from the rationale on which it is based.

\subsection{Implementation for the testing center}

In order to implement the EEPAS and PPE models for the Japan testing center, the continuous rate densities of these models are integrated over the latitude, longitude, magnitude and time limits of each cell to compute the expected number of earthquakes. The integration is performed by averaging the rate densities over an inner grid of $3^{4}$ points regularly spaced in time, magnitude, latitude and longitude within each cell. This discretization into cells is bound to affect the performance of the models to some extent, but the cell sizes are small enough so that the effects on perfor- mance should be quite small.

Figure 9 shows the spatial distribution of the normalised cell expectations for the three-month period April-June 2009 for the EEPAS model at magnitudes 5.0, 6.0 and 7.0 and the PPE model at magnitude 5.0. The expectations are normalised relative to a reference (RTR) model in which one earthquake per year is expected exceeding magnitude $m$ in an area of $10^{m} \mathrm{~km}^{2}$. For the PPE model, the spatial distribution is independent of magnitude for magnitudes greater than 5. For the EEPAS model, the spatial distribution varies with magnitude depending on the times of occurrence, magnitude and location of recent earthquakes. The variation with time is only appreciable over a year or more. Examples showing the variation of EEPAS model forecasts with time were given by Rhoades and Evison (2004, 2005). For a given set of EEPAS parameters, the higher the magnitude, the more slowly the forecast varies. However, the values of the parameters $a_{T}$ and $b_{T}$ for the lower magnitude classes (Table 1) are such that the forecasts hardly vary any faster for magnitudes below 5 than for magnitude 5 .

\section{Conclusion}

The EEPAS and PPE models have been submitted to the Japan Testing Center for testing in the Japan mainland region in the three-months and one-year forecast classes. The role of the PPE model here is that of a spatially-smoothed reference model, against which the performance of timevarying models such as EEPAS can be compared. 


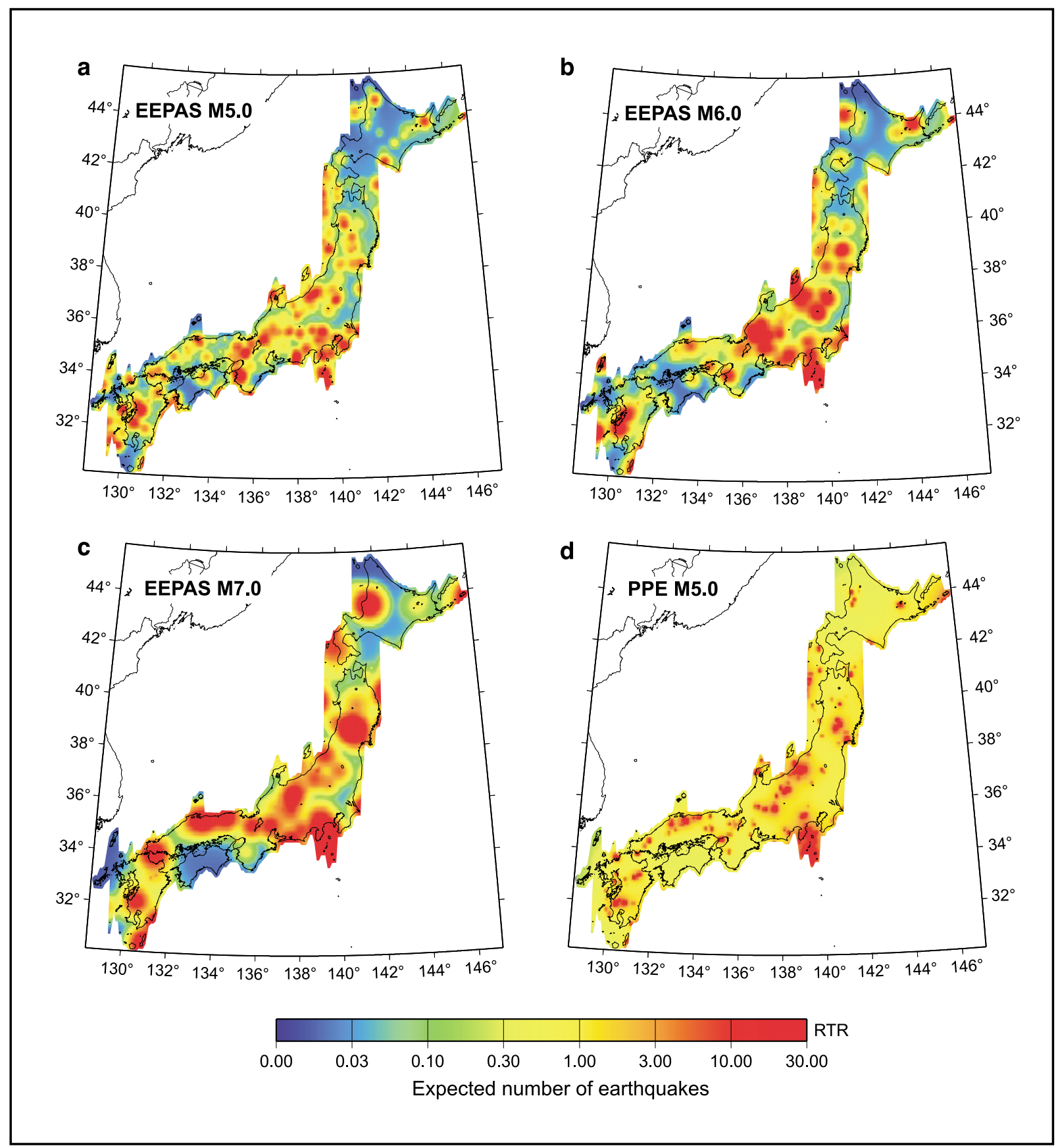

Fig. 9. Expected number of earthquakes for the period April-June 2009, in RTR units, i.e. normalized relative to a reference model in which one earthquake per year is expected above magnitude $m$ in an area of $10^{m} \mathrm{~km}^{2}$. (a) EEPAS model, $M$ 5.0; (b) EEPAS model, $M$ 6.0; (c) EEPAS model, $M 7.0$; (d) PPE model, $M$ 5.0.

The EEPAS model has never before been applied with a magnitude threshold as low as 4.0, which is the minimum magnitude for the three-month class. The adaptation of the model to such a low target magnitude has brought into focus some issues which cannot be resolved without further research. In particular, it has shown that the model needs further development to rigorously handle magnitudedependence in the proportion of aftershocks and in the $b$ value. Moreover, the unexplained differences in parameters of the EEPAS time and location distributions between target magnitude classes require further investigation. Also, the possibility of adapting the EEPAS model for testing in the wider Japan region can be considered. Further research into these matters is likely to result in one or more new models being submitted for testing in the future.

The EEPAS model forecasts could be applied in linear combination with one or more models from the one-day class to generate new one-day forecasting models (Rhoades and Gerstenberger, 2009). The performance of such hybrid models would help to clarify the relative information value of the precursory scale increase phenomenon with that of short-term clustering for forecasting over a range of target magnitudes. The analysis performed here suggests that the relative value of the precursory scale increase phenomenon should increase as the target magnitude is increased. 
Acknowledgments. This research was supported by the New Zealand Foundation for Research, Science and Technology under contract C050804 and by GNS Science Capability Funding. A. Christophersen, M. Stirling, W. Smith, M. Werner, E. Papadimitriou and editor K. Nanjo provided helpful reviews of the manuscript.

\section{References}

Akaike, H., A new look at the statistical model identification, IEEE Trans. Automatic Control, AC-19, 716-723, 1974.

Bäth, M., Lateral inhomogeneities of the upper mantle, Tectonophysics, 2 , 483-514, 1965.

Console, R. and M. Murru, A simple and testable model for earthquake clustering, J. Geophys. Res., 106, 8699-8711, 2001.

Evison, F. F. and D. A. Rhoades, The precursory earthquake swarm in Japan: hypothesis test, Earth Planets Space, 51, 1267-1277, 1999.

Evison, F. F. and D. A. Rhoades, Model of long-term seismogenesis, Ann. Geofis., 44(1), 81-93, 2001.

Evison, F. F. and D. A. Rhoades, Precursory scale increase and longterm seismogenesis in California and northern Mexico, Ann. Geophys., 45(3/4), 479-495, 2002.

Evison, F. F. and D. A. Rhoades, Demarcation and scaling of long-term seismogenesis, Pure Appl. Geophys., 161(1), 21-45, 2004.

Harte, D. and D. Vere-Jones, The entropy score and its uses in earthquake forecasting, Pure Appl. Geophys., 162(6/7), 1229-1253, 2005.

Imoto, M. and D. A. Rhoades, Seismicity models of moderate earthquakes in Kanto, Japan utilizing multiple predictive parameters, Pure Appl. Geophys., 167(6/7), 831-843, 2010.

Jackson, D. D. and Y. Y. Kagan, Testable earthquake forecasts for 1999, Seismol. Res. Lett., 70(4), 393-403, 1999.
Ogata, Y., Estimation of the parameters in the modified Omori formula for aftershock sequences, J. Phys. Earth, 31, 115-124, 1983.

Ogata, Y., Statistical models for standard seismicity and detection of anomalies by residual analysis, Tectonophysics, 169, 159-174, 1989.

Ogata, Y., Space-time point process models for earthquake occurrences, Ann. Inst. Stat. Math., 50, 379-402, 1998.

Rhoades, D. A., Application of the EEPAS model to forecasting earthquakes of moderate magnitude in southern California, Seismol. Res. Lett., 78(1), 110-115, 2007.

Rhoades, D. A., Long-range earthquake forecasting allowing for aftershocks, Geophys. J. Int., 178, 244-256, 2009.

Rhoades, D. A. and F. F. Evison, Long-range earthquake forecasting with every earthquake a precursor according to scale, Pure Appl. Geophys., 161(1), 47-71, 2004.

Rhoades, D. A. and F. F. Evison, Test of the EEPAS forecasting model on the Japan earthquake catalogue, Pure Appl. Geophys., 162(6/7), 1271$1290,2005$.

Rhoades, D. A. and F. F. Evison, The EEPAS forecasting model and the probability of moderate-to-large earthquakes in central Japan, Tectonophysics, 417(1/2), 119-130, 2006.

Rhoades, D. A. and M. C. Gerstenberger, Mixture models for improved short-term earthquake forecasting, Bull. Seismol. Soc. Am., 99(2A), 636-646, 2009.

Utsu, T., A statistical study on the occurrence of aftershocks, Geophys. Mag., 30, 521-605, 1961.

Zhuang, J., Y. Ogata, and D. Vere-Jones, Stochastic declustering of spacetime earthquake occurrence, J. Am. Stat. Ass., 97, 369-380, 2002.

D. A. Rhoades (e-mail: d.rhoades@gns.cri.nz) 